\title{
Parasite transmission: reconciling theory and reality
}

\author{
A. FENTON*, J. P. FAIRBAIRN*, R. NORMAN† and P. J. HUDSON* \\ *Institute of Biological Science, University of Stirling, Stirling, Scotland, FK9 4LA, UK; and † Department of \\ Computing Science and Mathematics, University of Stirling, Stirling, Scotland, FK9 4LA, UK
}

\section{Summary}

1. Arguably the most important and elusive component of host-parasite models is the transmission function. Considerable empirical and theoretical work has focused on determining the correct formulation of this function although, to date, there has been little attempt to combine these studies to develop general insights into how observed transmission rates affect host-parasite dynamics.

2. Here, estimates of transmission rates from a range of host-parasite systems in the literature are described using a phenomenological function which takes into account how transmission varies with host and parasite densities. This function is placed in the appropriate model framework to determine the consequences of the observed transmission rates for each system.

3. All of the parasites had decreasing per capita transmission rates with increasing parasite densities suggesting that parasites tend to saturate at high densities, either as hosts become limiting or due to heterogeneities amongst the host population. In terms of the responses to host density, the parasites fell into two groups: those with increasing or decreasing transmission rates. This dichotomy was due to the biology of the organisms; the former group infect through cannibalism, which increased at high densities as the individuals became stressed, whereas the latter group infected through free-living stages, resulting in a form of spatial structuring reducing the number of hosts available for infection.

4. A metapopulation model was developed where hosts and parasites interacted in discrete patches according to the appropriate transmission function, with neighbouring patches linked by dispersal. The model suggested that small-scale, localized transmission events can drive large-scale epizootics at the metapopulation level. This emphasizes the importance of correctly describing and quantifying the transmission function at the individual level.

5. Traditionally, the formulation of the transmission function has depended on the scale of observation. This work shows that transmission should be considered from the viewpoint of the organisms concerned. Observed transmission rates are a consequence of the biology of the individuals meaning it should be possible to develop a priori hypotheses concerning the nature of the transmission function from a basic understanding of the life history of the organisms concerned.

Key-words: microparasite, non-linear transmission, parasite persistence, spatial model.

Journal of Animal Ecology (2002) 71, 893-905

\section{Introduction}

Following the pioneering work of Anderson \& May (1979, 1981) and May \& Anderson (1979), a great number of studies have been carried out with the intention of understanding the mechanisms underlying the stability of host-parasite interactions. This progression

(C) 2002 British

Ecological Society
*Correspondence: Tel. 01786 467819. Fax: 01786 464994. Email: acf1@stir.ac.uk of work has led to the refinement of many of the assumptions of the original models and a great deal of headway has been made in determining the factors that influence parasite persistence. One of the major assumptions of the original models that has received considerable attention is that of mass action transmission (Liu, Hethcote \& Levin 1987; Hochberg 1991; Begon et al. 1999; McCallum, Barlow \& Hone 2001). This represents one of the most important components of host-parasite models, the transmission process itself, 
and states that hosts and parasites mix randomly such that the per capita transmission rate is independent of the density of both susceptible hosts and infectious units.

Despite the traditional assumptions of constant transmission rates, it has been shown empirically for a number of parasite systems that transmission rates are not independent of the density of hosts and/or infectious units (Dwyer \& Elkinton 1993; D'Amico et al. 1996; Knell, Begon \& Thompson 1996; Dwyer, Elkinton \& Buonaccorsi 1997; Knell, Begon \& Thompson 1998a). Furthermore, a number of workers have shown theoretically that different formulations of the transmission function can drastically affect the dynamics and stability of host-parasite systems (Liu et al. 1987; Hochberg 1991; Briggs \& Godfray 1995; Dwyer et al. 2000). Although the observed transmission rates are likely to have important consequences for the dynamics of host-parasite systems, little attempt has been made to combine these empirical studies and develop any coherent, general rules concerning how realistic transmission functions affect host-parasite dynamics across a range of systems.

There is clearly a need to develop a more integrated approach, due to the apparent confusion in the interpretation of the transmission process. Laboratorybased or small-scale field experiments, which consider transmission close to the individual scale, have looked for non-linearities in the transmission rate with changing densities (Dwyer \& Elkinton 1993; D'Amico et al. 1996; Knell et al. 1996, 1998a). Conversely, the theoreticians using population-based models have tended to categorize transmission as being either frequency- or density-dependent and this distinction has been supported by field data obtained by population-level timeseries analysis (Begon et al. 1998, 1999). However, it has been argued previously that it is the transmission events at the individual level that are the main driving force behind epizootics seen at the metapopulation level (Dwyer \& Elkinton 1993). It may be that our understanding of transmission and, specifically, the choice of transmission function in the past has been due as much to the scale of observation as to the biology of the organisms concerned.

The aim of the present paper is twofold: first, to combine empirically derived estimates of transmission rate from the literature with the appropriate hostmicroparasite models to assess the effects of realistic formulations of the transmission function on parasite persistence; and secondly, to increase the scale of our model and assess whether these localized transmission events can indeed explain large-scale phenomena of the host-parasite system at the metapopulation level. In particular, we ask: what functional forms of transmission lead to persistence of a host-microparasite system with free-living infective stages? Are there any general patterns of transmission biology that emerge for different parasite guilds? What are the implications of different transmission functions for parasite persistence at the metapopulation level?

\section{Methods}

\section{THEORETICAL CONSIDERATIONS}

\section{Generic host-microparasite model with free-living infective stages}

The majority of empirical studies of parasite transmission dynamics use microparasites with free-living infective stages infecting insect hosts, so we will use the following modified version of the general invertebrate host-microparasite model with free-living infective stages, based on the Anderson \& May (1981) model G:

$$
\begin{aligned}
& \frac{d \mathrm{X}}{d t}=A \mathrm{X}+B \mathrm{Y}-f(\mathrm{X}, \mathrm{P}) \\
& \frac{d \mathrm{Y}}{d t}=f(\mathrm{X}, \mathrm{P})-C \mathrm{Y} \\
& \frac{d \mathrm{P}}{d t}=\Lambda \alpha \mathrm{Y}-\mu \mathrm{P}-f(\mathrm{X}, \mathrm{P})
\end{aligned}
$$

$\mathrm{X}$ is the density of susceptible hosts, $\mathrm{Y}$ is the density of infected hosts and $\mathrm{P}$ is the density of free-living infective stages. $A$ is the net rate of increase of the susceptible population due to birth from susceptibles, $r_{1}$, and background mortality, $b\left(A=r_{1}-b\right)$. The susceptible population is also increased through reproduction from infecteds, $r_{2}$, and recovery, $\gamma\left(B=r_{2}+\gamma\right)$. Because we are dealing with invertebrate hosts, we assume there is no life-long immunity to infection following recovery; hosts are either susceptible or infected. Infecteds that do not recover die either due to parasitism at rate $\alpha$ or through natural mortality at rate $b$. Hence, infecteds are lost at a net rate $C(=\gamma+\alpha+b)$. At death, infecteds release $\Lambda$ infective juveniles into the environment, which die at a constant rate $\mu$. Infection occurs at rate $f(\mathrm{X}, \mathrm{P})$ which, in the simplest case is assumed to be directly proportional to parasite and host densities at a constant, per capita transmission rate $\beta$ such that $f(\mathrm{X}, \mathrm{P})=\beta \mathrm{XP}$.

\section{Incorporating non-linear transmission rates}

The transmission function described above $[f(\mathrm{X}, \mathrm{P})=$ $\beta \mathrm{XP}]$ is linear in both $\mathrm{X}$ and $\mathrm{P}$ and corresponds to the mass action assumption traditionally used by most host-parasite models. However, a number of theoretical approaches have been proposed to introduce nonlinearities into the transmission function (Liu et al. 1987; Hochberg 1991; Heesterbeek \& Metz 1993; Antonovics, Iwasa \& Hassell 1995). Because we are interested in comparing across a variety of systems, we require a simple and flexible formulation of non-linear transmission that makes no assumptions about the underlying mechanisms leading to observed transmission rates. Of the different formulations available, the phenomenological function adopted by Hochberg (1991) is arguably the most parsimonious and flexible 
Unifying parasite transmission concepts
(C) 2002 British

Ecological Society, Journal of Animal Ecology, 71, 893-905
(Dwyer et al. 1997; McCallum et al. 2001), allowing the effects of both host and parasite density to be incorporated easily through the addition of two extra parameters. Using a simple, directly transmitted microparasite model, Hochberg (1991) replaced the per capita transmission rate, $\beta$ with:

$\beta=\beta^{\prime} \mathbf{S}^{p} \mathbf{I}^{q}$

eqn 4

where $\beta^{\prime}$ is the rescaled transmission parameter and $p$ and $q$ are constants representing how the densities of susceptibles (S) and infecteds (I) independently affect the per capita transmission rate. Clearly, if $p$ and $q$ are 0 , mass action transmission is recovered. To apply Hochberg's approach to the microparasite system with free-living infective stages, we replace $f(\mathrm{X}, \mathrm{P})$ in equations $1-3$ with $\beta^{\prime}\left(\mathrm{X}^{p} \mathrm{P}^{q}\right) \mathrm{XP}$.

\section{STUDY SYSTEMS}

By using this model framework and the empirically derived estimates of transmission from the literature we can assess how observed transmission rates affect the stability of a range of host-microparasite systems. For ease of comparison, we concentrated on studies in which the per capita transmission rate, $\beta$, has been estimated for a range of host and/or parasite densities under controlled conditions rather than larger, fieldbased studies. In all cases, the experimental design is similar (see Appendix A for details); varying numbers of hosts and infectious units (either the infective stages of the parasites or infected hosts) are placed into an arena of fixed size for a period of time, short enough to minimize the probability that either host or parasite numbers change due to background mortality or reproduction. Given the number of hosts surviving at the end of the experiment, the authors use a simplified version of a population model (similar to that in equations 1-3) to obtain estimates of $\beta$ at each combination of host and parasite density. The manner in which $\beta$ changes with host and parasite density allows insight into the shape of the transmission function and shows whether it obeys the linear, mass action assumption. In the studies selected here, the authors presented their data in the form of $\beta$ against density and, using leastsquares non-linear regression we fit equation 4 to these data to obtain estimates of $\beta^{\prime}, p$ and $q$ for each study. Ideally it would be preferable to fit equation 4 directly to the raw data for each experiment. However, these data are summarized by the authors in each study to produce their estimates of $\beta$ and so considerable variation may be lost by relying on the data in this form. Clearly, our findings can only be as good as the data presented and, while we acknowledge that for some of the studies the data are sparse, we believe this approach is valid to give a general, cross-species description of the trends in how transmission varies with host and parasite densities. In this section we describe the study systems and experimental designs; details of the equations that the authors used to calculate the transmission coefficient are relegated to Appendix A.

Nuclear polyhedrosis virus infecting Lymantria dispar Linnaeus (Lepidoptera: Lymantriidae): D'Amico et al. (1996)

D'Amico et al. (1996) used mesh bags to enclose oak branches into which they placed a varying number of gypsy moth, $L$. dispar larvae (20 or five) infected with nuclear polyhedrosis virus and a fixed number of susceptible, uninfected larvae (25). The bags were left for 7 days before the previously uninfected larvae were removed to determine whether they had become infected. D'Amico et al. (1996) incorporated a term for the decay rate of the virus particles $(\mu)$ and estimated the transmission parameter by fitting equation $\mathrm{A} 2$ to their data (Appendix A). The experiment was replicated 16 times. Note that D'Amico et al. (1996) varied only the initial number of infected larvae, not the initial number of susceptible larvae, so it is only possible to estimate $q$ from the estimates of $\beta$ for this experiment. However, D'Amico et al. (1996) also re-interpreted data of Woods \& Elkinton (1987) and Dwyer \& Elkinton (1993), showing how transmission rate varies with susceptible and infected host densities in a naturally occurring population. We are therefore able to estimate values for $\beta^{\prime}, p$ and $q$ from these estimates of $\beta$.

Bacillus thuringiensis infecting Plodia interpunctella Hubner (Lepidoptera: Pyralidae): Knell et al. (1996)

Knell et al. (1996) carried out a number of laboratory experiments using the Indian meal moth, $P$. interpunctella to determine how B. thuringiensis transmission rates varied with density of infected and susceptible hosts. They varied the initial density of susceptible hosts from five to 20 , challenged with two infectious cadavers and then varied the initial density of infectious cadavers from two to 10 , challenging 10 uninfected larvae; the experiments were run for $12 \mathrm{~h}$. The experiment was repeated for both 4 th and 5 th instar larvae and the transmission coefficient estimated using equation A1. The experimental design enables us to estimate values of $\beta^{\prime}, p$ and $q$ from the entire data set.

Nuclear polyhedrosis virus and $\mathrm{L}$. dispar:

Dwyer et al. (1997)

Dwyer et al. (1997) carried out a similar experiment to that of D'Amico et al. (1996), using a variable number of infected larvae (two up to 75) and 25 susceptible larvae within each bag, run for 7 days. The experiment was conducted for 3 years, replicated four times in 1994 and eight times in 1995 and 1996 and for two host strains (laboratory and feral), although we present only the data for the feral strains here. The data were presented as 'transmission' $\left(-\ln \left[\mathrm{X}_{\mathrm{t}} / \mathrm{X}_{0}\right]\right)$ against virus density, so that the per capita transmission coefficient is 
this transmission value divided by $\mathrm{P}_{0}$. Once again, only the density of infecteds was varied, so only $q$ can be estimated from the data.

Granulosis virus ( PiGV) infecting P. interpunctella: Knell et al. (1998a)

Knell et al. (1998a) carried out similar experiments to those of Knell et al. (1996), using a granulosis virus to infect $P$. interpunctella. First, they varied the number of susceptible hosts from five to 20, challenged with two infectious cadavers and secondly they varied the initial number of infectious cadavers from one to four, challenging 10 uninfected larvae. The experiments were replicated eight times. The transmission coefficient was then estimated for each combination of densities from equation A1. Once again, the experimental protocol enables us to estimate $\beta^{\prime}, p$ and $q$ from the entire data set.

Nuclear polyhedrosis virus infecting Malacosoma californicum pluviale (Dyar) (Lepidoptera:

Lasiocampidae): Beisner \& Myers (1999)

Beisner \& Myers (1999) conducted field trials similar to D'Amico et al. (1996), setting up colonies of varying densities $(32,64,128$ or 256) of the western tent caterpillar, $M$. californicum pluviale on oak trees and inoculating with varying densities $(10,20$ or 40$)$ of infected larvae. The trial was replicated five times and run for 9 days. The transmission coefficient was calculated for each combination using equation $\mathrm{A} 1$ and the presented estimates of $\beta$ allow us to calculate $\beta^{\prime}, p$ and $q$.

Steinernema feltiae Filipjev (Nematoda: Rhabditida) infecting Galleria mellonella (Linnaeus) (Lepidoptera: Pyralidae): Fairbairn et al. (unpublished)

We have previously measured the effect of different densities of the nematode $S$. feltiae and host, the greater waxmoth $G$. mellonella, on the per capita transmission rate, $\beta$ (Fairbairn et al., unpublished). The experiment was conducted in sand-filled pots, where four densities of both host and nematode were used in a factorial design so that each host density was challenged by each nematode density. After $24 \mathrm{~h}, \beta$ was calculated for each combination of host and nematode density using equation A3. The experiment was replicated six times for each combination of host and nematode densities and, because a factorial design was used, we are able to estimate $\beta^{\prime}, p$ and $q$ from the data presented.

Schistosoma mansoni Sambon (Trematoda: Digenea) infecting Biomphalaria glabrata Say (Gastropoda:
For comparison, we include data from one laboratory study into transmission rates using a macroparasite; $S$. mansoni miracidia infecting the snail intermediate host,
B. glabrata. While the model presented in equations 1-3 cannot be used to describe the full host-macroparasite system, due to the need to consider the distribution of parasites per host, comparisons of the shape of the transmission function can still be made as this is unaffected by the reproductive biology of the parasite following infection. Varying numbers of uninfected snails (one replicated 32 times, three replicated 16 times and five, seven and nine replicated eight times) were each challenged with varying numbers of miracidia (120 ) in fixed arenas. The snails were exposed to infection for $3 \mathrm{~h}$, after which they were crushed and examined for larval schistosomes. The transmission coefficient was calculated using equation A4 and the experimental protocol enables $\beta^{\prime}, p$ and $q$ to be estimated from the entire data set.

\section{CONSEQUENCES OF NON-LINEAR \\ TRANSMISSION RATES AT THE METAPOPULATION LEVEL}

Dwyer \& Elkinton (1993) argued that the main determinant of epizootics in the field may be the small-scale transmission events and that it is often possible to deduce large-scale phenomena of epizootics from smallscale measurements of transmission. As mentioned previously, the transmission experiments discussed here were all conducted in small, fixed-size arenas, which can be thought of as representing localized patches where subpopulations of hosts and parasites closely interact. To assess the relative contributions of spatial structure and localized transmission events and to determine whether these small-scale interactions can drive large-scale phenomena, we develop a spatially explicit model, joining a number of these localized patches together into a larger network, or metapopulation, linked by local dispersal events between neighbouring patches. The effects of nonlinear transmission rates have not been explored previously in a spatially explicit context and this analysis provides insight into how transmission and dispersal interact to influence dynamics at the metapopulation level.

\section{Metapopulation model with linear transmission rates}

Initially, we deal solely with linear transmission functions to determine the effects of dispersal rates on system stability; the next section deals with the effect of non-linear transmission rates. There are a number of approaches that may be adopted to consider hostparasite interactions in space. For the purposes of this analysis, we adopt a modified coupled map lattice framework where the landscape comprises a square grid of small patches (Comins, Hassell \& May 1992; Rohani \& Miramontes 1995; Comins \& Hassell 1996; White, Begon \& Bowers 1996). Within each patch the hosts and parasites undergo a period of interaction, as described by equations $1-3$, following which a fixed 
Unifying parasite transmission concepts
(C) 2002 British

Ecological Society, Journal of Animal Ecology, 71, 893-905 proportion of hosts $\left(D_{H}\right)$ and parasites $\left(D_{P}\right)$ disperse, distributing themselves equally to the surrounding eight patches before the next period of interaction. This localized, diffusive movement across the landscape has been shown to have a stabilizing effect on parasitoid and microparasite models (Comins \& Hassell 1996; White et al. 1996).

For the majority of systems examined here, the pathogen is invariably lethal, killing host larvae before development to adulthood, so we describe the interaction of host and parasite within each local patch by using equations 1-3 with no infected reproduction or recovery $\left(r_{2}=\gamma=0\right)$ and linear transmission rates $[f(\mathrm{X}, \mathrm{P})=$ $\beta \mathrm{XP}]$. For the results presented, each period of interaction was assumed to be 14 days, although a range of simulations was carried out where this period of interaction was varied from 1 to 30 days and it was found not to affect the general predictions of the model. For simplicity, we make the assumption that the host population is not age-structured, thereby removing the need to consider bouts of short-scale host movements (e.g. by the juvenile hosts) followed by long-range dispersal (by the adult hosts). The model is run for 300 days, to approximate a single year of interaction, which is sufficient time to determine whether the host and parasites establish a state of coexistence, or whether either species goes extinct.

\section{Metapopulation model with non-linear transmission rates}

Finally, we combine the work in the preceding sections to determine how non-linear transmission rates and explicit spatial structure interact to influence the persistence of host-parasite systems. In this section we assume transmission rates within each patch of the coupled map lattice depend on local host and parasite density as described in equation $4[f(\mathrm{X}, \mathrm{P})=$ $\left.\beta^{\prime}\left(\mathrm{X}^{p} \mathrm{P}^{q}\right) \mathrm{XP}\right]$.

\section{Results}

\section{THEORETICAL CONSIDERATIONS}

A full stability analysis has previously been carried out on models similar to that in equations 1-3 with linear, mass action transmission (Anderson \& May 1981; Dwyer 1994; Boots 1999) but we repeat much of the analysis in Appendix B for completeness. An important finding is that if host and pathogen are to persist at a stable equilibrium it is essential for there to be some form of increase in the susceptible population from the infected population, either through recovery or reproduction (Appendix B; Anderson \& May 1981; Dwyer 1994; Boots 1999). However, as the pathogen is invariably lethal for the majority of the systems examined here, it is unlikely that host and parasite would be expected to persist without the addition of some other stabilizing factor.
Stability analysis of equations 1-3 with non-linear transmission rates (see Appendix C) reveals two boundaries separating the stable and unstable regions of parameter space, corresponding to those found by Hochberg (1991) for his directly transmitted model (Fig. 1). The analysis also reveals an additional, nonlinear boundary which simulations show to separate the region of stable regulation from that of stable limit cycles. As a general rule, the present model is less stable than the directly transmitted parasite model of Hochberg (1991) but regions that are stable in the current model are also stable in the corresponding Hochberg model.

The first criterion for stability is the same regardless of the specific life history details of the model and is given by the inequality $p+q>-1$, equivalent to $\mathrm{R}_{0}>1$, indicating that stability is determined, at least in part, by the total response of both host and parasite combined $(p+q)$, regardless of the magnitudes of the individual responses. Therefore, as Hochberg (1991) found, if the per capita transmission rate increases with the density of susceptible hosts $(p>0)$ the system is likely to be stable (Fig. 1). Below the line $p+q=-1$ the pathogen is unable to regulate the host population, which grows exponentially.

The location of the second stability boundary depends on the details of the host-parasite system. In general, stability is more likely if parameter values favour the increase of susceptible hosts. For instance, increasing $r_{1}, r_{2}$ or $\gamma$ (all of which increase the number of susceptibles) tends to increase the region of stability and increase the viability of parasites with increasing responses of transmission to parasite density (i.e. the region of stability where $q>0$ ) (Fig. 1a-d). In addition, increasing $r_{2}$ and $\gamma$ leads to an increase in the viability of systems with decreasing responses of transmission to susceptible host density (i.e. the region of stability where $p<0$ ). Finally, increasing the mortality rate of the free-living stages $(\mu)$ tends to reduce the region of stability and decrease the viability of systems with increasing responses of transmission to parasite density (i.e. the region of stability where $q>0$ ) (Fig. 1e,f). It should be emphasized that the addition of non-linear transmission rates and, in particular, positive $p$-values and intermediate $q$-values can stabilize the hostpathogen model in equations $1-3$ even in the absence of recovery or reproduction by infecteds $\left(\gamma=r_{2}=0\right)$.

\section{COMBINATION OF THEORY AND DATA}

Having shown that non-linear transmission rates can, in theory, stabilize host-parasite systems, the question remains: do they? What is the functional form of transmission and where do these parasite systems sit in Fig. 1? To answer these questions we used non-linear, least squares regression to fit equation 4 to the estimates of $\beta$ from the data sets in the literature and determined best-fit values for $p, q$, and $\beta^{\prime}$. By locating the resulting parameter values in the model framework 
898

A. Fenton et al. (a)

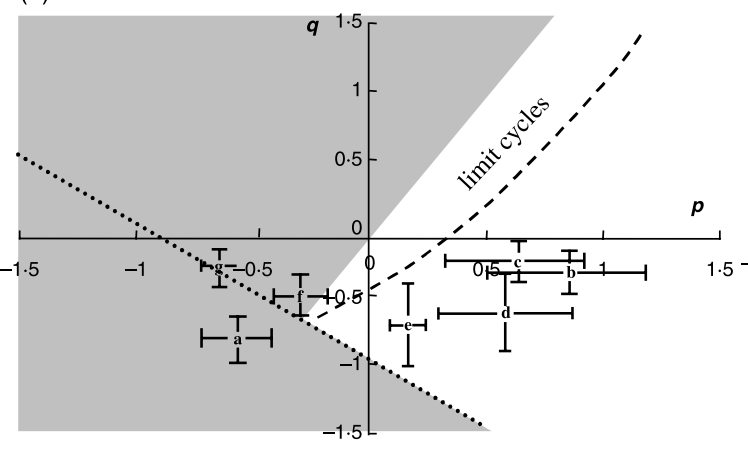

(c)

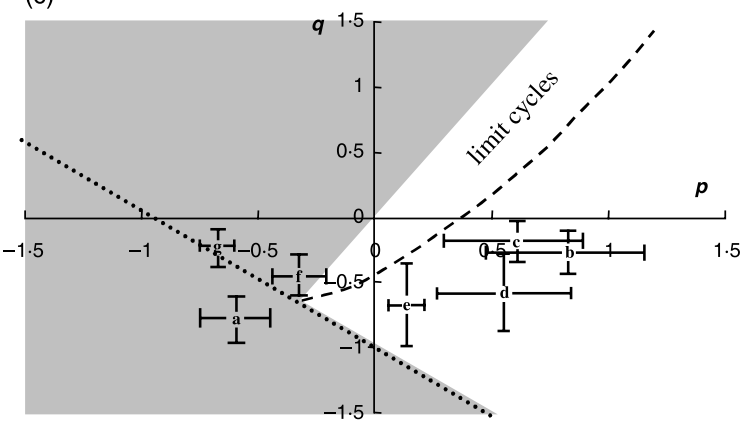

(b)

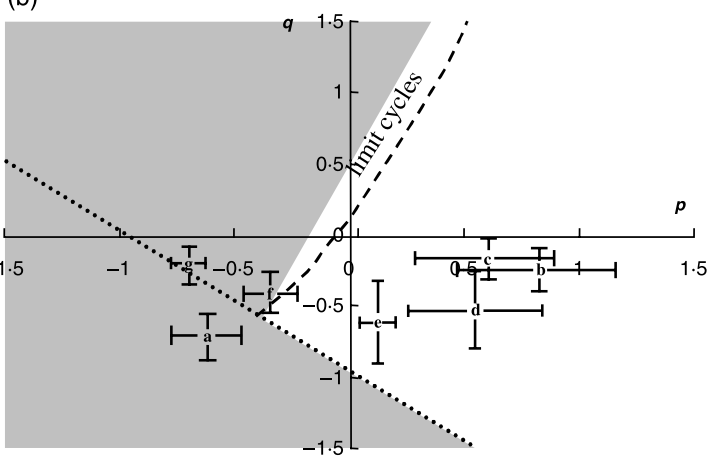

(d)

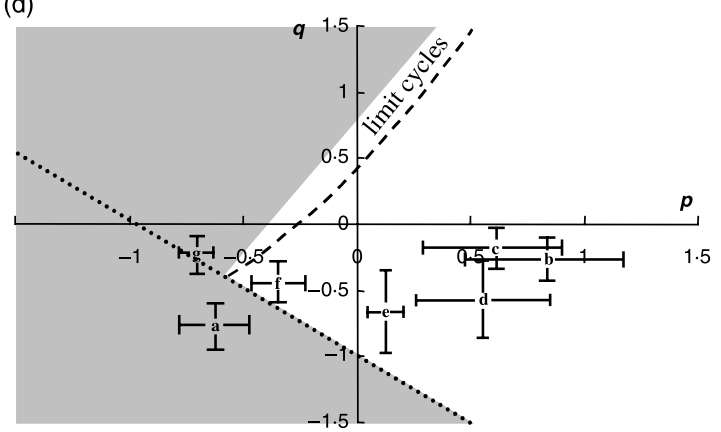

(f)

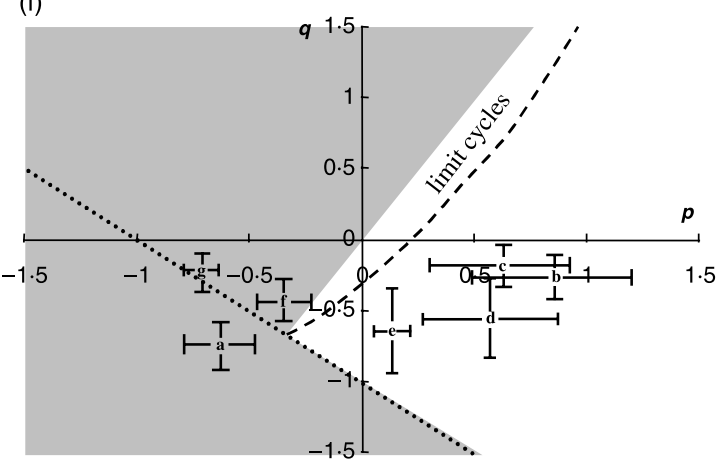

(e)

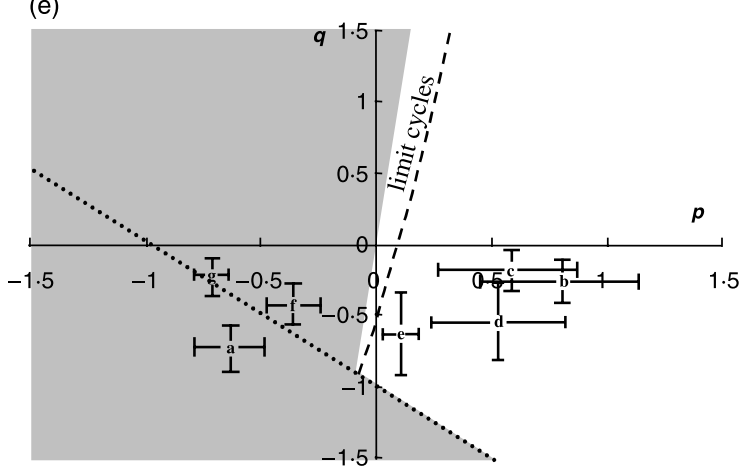

Fig. 1. $p-q$ parameter space for the insect-host microparasite model where the transmission function is $f(\mathrm{X}, \mathrm{P})=\beta^{\prime} \mathrm{X}^{p+1} \mathrm{P}^{q+1}$. The unshaded region shows stable host-parasite persistence, whereas the shaded region represents unstable host-parasite interaction. The dotted line shows the boundary $p+q=-1$, equivalent to $\mathrm{R}_{0}=1$. Unless otherwise stated, parameter values are $r_{1}=0 \cdot 2, r_{2}=0$, $\gamma=0, b=0 \cdot 1, \alpha=0 \cdot 1, \Lambda=10000, \mu=0 \cdot 1, \beta^{\prime}=0 \cdot 0005$. (a) $r_{2}=0$ (b) $r_{2}=0 \cdot 04$. (c) $\gamma=0$ (d) $\gamma=0 \cdot 4$. (e) $\mu=0 \cdot 01$ (f) $\mu=0 \cdot 1$. The letters on the figures (with standard error bars) show the location of the parameter estimates from the following systems: $(a)$ NPV $-L$. dispar (D'Amico et al. 1996), (b) B. thuringiensis-4th instar P. interpunctella (Knell et al. 1996), (c) B. thuringiensis-5th instar $P$. interpunctella (Knell et al. 1996), (d) PiGV-P. interpunctella (Knell et al. 1998a), (e) NPV-M. californicum pluviae (Beisner \& Myers 1999), ( $f$ ) S. feltiae-G. mellonella (Fairbairn et al., unpublished) (g) S. mansoni-B. glabrata (Carter et al. 1982).

(C) 2002 British

Ecological Society, Journal of Animal Ecology, 71, 893-905 described above, we can determine if the observed transmission rates are non-linear and how they influence system stability.

While it is difficult to make specific comments about each system without accurately parameterizing the appropriate model, it is possible to make some general conclusions about the probable consequences of the observed transmission rates on stability. All the systems considered here have decreasing responses of transmission rate to parasite density $(q<0)$ (Table 1; Fig. 1) suggesting that there is an upper limit to the rate of transmission regardless of how great the density of infectious units. In terms of the response of transmission to the density of susceptibles, there appear to be two distinct groups: those with positive responses (B. thuringiensis and PiGV infecting P. interpunctella and NPV infecting $M$. californicum pluviale) and those with negative responses (all others including the schistomosome macroparasite system) (Table 1; Fig. 1). The parasites with positive $p$-values are all situated above the $p+q=-1$ line (which is constant, regardless of specific model details) and below the $q=0$ line. Therefore, regardless of the details of the model (including the distinction between directly transmitted parasites as assumed by Hochberg (1991) and those transmitted via free-living stages as assumed here), these systems are situated firmly in the stable region of parameter space. 
899

Unifying parasite transmission

concepts
(C) 2002 British

Ecological Society, Journal of Animal Ecology, 71, 893-905

Table 1. Summary of the estimated $\beta^{\prime}, p$ and $q$-values (with standard errors) and $R^{2}$ values obtained by a non-linear least-squares fit of equation 4 to the data sets presented. In studies marked with an asterisk, the infectious unit was the individual free-living infective particle, whereas the remaining studies used the infected host cadaver

\begin{tabular}{|c|c|c|c|c|c|c|}
\hline Host & Parasite & $\beta^{\prime}$ & $p$ & $q$ & $R^{2}$ & Reference \\
\hline L. dispar & NPV & $\begin{array}{l}3 \cdot 7 \times 10^{-12} \mathrm{~m}^{2} \text { day }^{-1} \\
\left(4.53 \times 10^{-13}\right)\end{array}$ & - & $\begin{array}{r}-0 \cdot 622 \\
(0 \cdot 07)\end{array}$ & $0 \cdot 98$ & *D'Amico et al. (1996) \\
\hline L. dispar & NPV & $\begin{array}{l}3.0 \times 10^{-13} \mathrm{~m}^{2} \text { day }^{-1} \\
\left(3.55 \times 10^{-14}\right)\end{array}$ & $\begin{array}{c}-0 \cdot 598 \\
(0 \cdot 150)\end{array}$ & $\begin{array}{c}-0 \cdot 811 \\
(0 \cdot 136)\end{array}$ & $0 \cdot 84$ & *D'Amico et al. $\left(1996^{\mathrm{a}}\right)$ \\
\hline 4th instar $P$. interpunctella & B. thuringiensis & $\begin{array}{l}0.007 \text { area }^{-1} \\
(0 \cdot 007)\end{array}$ & $\begin{array}{l}0 \cdot 810 \\
(0 \cdot 365)\end{array}$ & $\begin{array}{r}-0 \cdot 284 \\
(0 \cdot 261)\end{array}$ & $0 \cdot 57$ & Knell et al. (1996) \\
\hline 5th instar $P$. interpunctella & B. thuringiensis & $\begin{array}{l}0.051 \text { area }^{-1} \\
(0 \cdot 045)\end{array}$ & $\begin{array}{c}0 \cdot 556 \\
(0 \cdot 310)\end{array}$ & $\begin{array}{c}-0 \cdot 202 \\
(0 \cdot 200)\end{array}$ & $0 \cdot 53$ & Knell et al. (1996) \\
\hline L. dispar 1994 & NPV & $\begin{array}{l}0 \cdot 024 \mathrm{~m}^{2} \mathrm{day}^{-1} \\
(0 \cdot 0046)\end{array}$ & - & $\begin{array}{c}-0 \cdot 382 \\
(0 \cdot 154)\end{array}$ & $0 \cdot 68$ & Dwyer et al. (1997) \\
\hline L. dispar 1995 & NPV & $\begin{array}{l}1.394 \mathrm{~m}^{2} \text { day }^{-1} \\
(0 \cdot 487)\end{array}$ & - & $\begin{array}{c}-0 \cdot 325 \\
(0 \cdot 148)\end{array}$ & $0 \cdot 64$ & Dwyer et al. (1997) \\
\hline L. dispar 1996 & NPV & $\begin{array}{l}2 \cdot 290 \mathrm{~m}^{2} \mathrm{day}^{-1} \\
(0 \cdot 502)\end{array}$ & - & $\begin{array}{c}-0.512 \\
(0.086)\end{array}$ & $0 \cdot 95$ & Dwyer et al. (1997) \\
\hline P. interpunctella & PiGV & $\begin{array}{l}0 \cdot 117 \text { area }^{-1} \\
(0 \cdot 088)\end{array}$ & $\begin{array}{l}0 \cdot 526 \\
(0 \cdot 305)\end{array}$ & $\begin{array}{c}-0.60 \\
(0 \cdot 29)\end{array}$ & $0 \cdot 59$ & Knell et al. (1998b) \\
\hline M. californicum & NPV & $\begin{array}{l}0.003 \text { area day }^{-1} \\
(0 \cdot 021)\end{array}$ & $\begin{array}{l}0 \cdot 210 \\
(0 \cdot 164)\end{array}$ & $\begin{array}{c}-0 \cdot 734 \\
(0 \cdot 280)\end{array}$ & $0 \cdot 48$ & Beisner \& Myers (1999) \\
\hline G. mellonella & S. feltiae & $\begin{array}{l}0 \cdot 145 \mathrm{~m}^{2} \mathrm{~h}^{-1} \\
(0 \cdot 086)\end{array}$ & $\begin{array}{c}-0 \cdot 304 \\
(0 \cdot 082)\end{array}$ & $\begin{array}{c}-0 \cdot 482 \\
(0 \cdot 127)\end{array}$ & $0 \cdot 74$ & *Fairbairn et al., unpublished \\
\hline B. glabrata & S. mansoni & $\begin{array}{l}0 \cdot 196 \text { litre }^{-1} \\
(0 \cdot 009)\end{array}$ & $\begin{array}{l}-0 \cdot 670 \\
(0 \cdot 042)\end{array}$ & $\begin{array}{r}-0.329 \\
(0.035)\end{array}$ & 0.92 & *Carter et al. (1982) \\
\hline
\end{tabular}

${ }^{a}$ Field data of Woods \& Elkinton (1987), for which transmission coefficients were estimated by Dwyer \& Elkinton (1993).

The stability of the parasites with negative $p$-values is more in doubt, although the NPV-L. dispar system studied by D'Amico et al. (1996) sits below the $p+$ $q=-1$ line (point $a$, Fig. 1), suggesting this pathogen is always unable to persist without some other stabilizing factor. In addition, the entomopathogenic nematode system, for which the model was specifically parameterized, sits in the unstable region of parameter space when there is no recovery or reproduction by infecteds (point $f$, Fig. 1) implying that non-linear transmission rates are insufficient to stabilize the nematode-host interaction. Simulations of equations $1-3$ with linear transmission rates $[f(\mathrm{X}, \mathrm{P})=\beta \mathrm{XP}]$ show that the host nematode interaction is highly unstable, displaying wild fluctuations in abundance (Fig. 2a). However, simulations of the non-linear transmission model incorporating the appropriate parameter estimates show that the fluctuations in numbers are even greater, due to the addition of a destabilizing force to an already unstable system (Fig. 2b). Therefore, although non-linear transmission rates can have a stabilizing influence on hostparasite dynamics, for entomopathogenic nematodes at least, they cannot explain the long term persistence of the parasite. It is difficult to make comparisons of the values of the rescaled transmission parameter $\beta^{\prime}$, since the various studies used different interpretations of the infectious unit (infected cadaver vs. virus particle), different time periods and different areas over which the experiment was conducted so the units of $\beta$ will vary between studies.

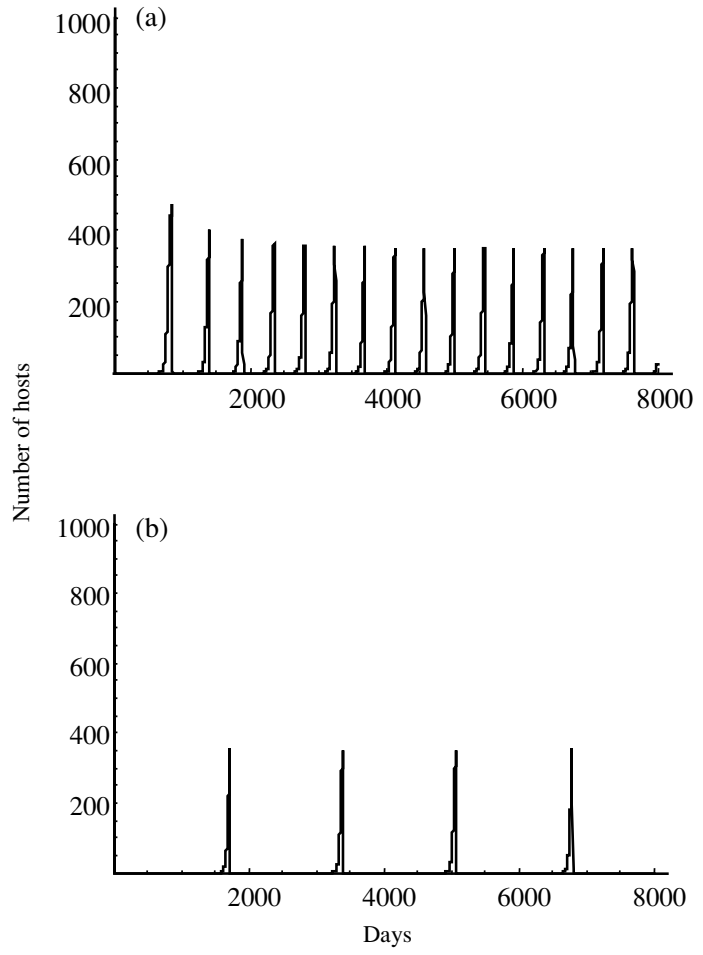

Fig. 2. Numerical simulations of equations 1-3 with no infected reproduction or recovery parameterized for the entomopathogenic nematode system. (a) linear transmission rates $[f(\mathrm{X}, \mathrm{P})=\beta \mathrm{XP}]$ and $(\mathrm{b})$ non-linear transmission rates $\left[f(\mathrm{X}, \mathrm{P})=\beta^{\prime} \mathrm{X}^{p+1} \mathrm{P}^{q+1}\right] . r_{1}=0 \cdot 2, r_{2}=0, \gamma=0, b=0 \cdot 1, \alpha=0 \cdot 08$, $\Lambda=10000, \mu=0 \cdot 5, \beta=0 \cdot 04, \beta^{\prime}=0 \cdot 145, p=-0 \cdot 304, q=$ $-0 \cdot 482$. 


\section{0}

A. Fenton et al.

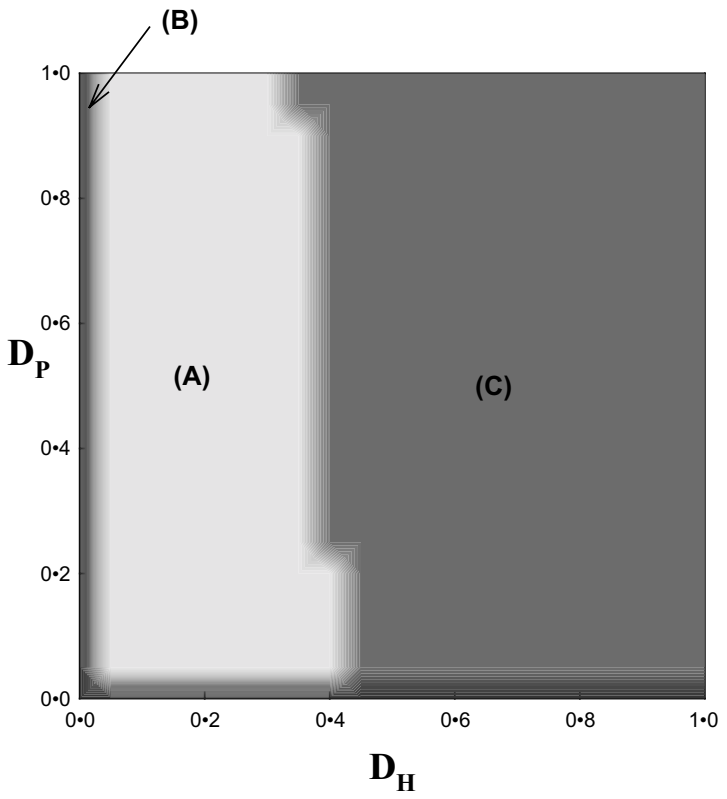

Fig. 3. The effect of different dispersal rates of the hosts $\left(D_{H}\right)$ and parasites $\left(D_{\mathrm{P}}\right)$ on the stability of the host-parasite metapopulation as obtained by numerical simulations of the model described in section $\mathrm{C} 1$ with linear transmission rates. Region A represents stable host-parasite persistence, B represents regions where the parasite becomes extinct and $\mathrm{C}$ represents regions where the host becomes extinct. $r_{1}=0 \cdot 1$, $r_{2}=0, \gamma=0, b=0 \cdot 1, \alpha=0 \cdot 08, \Lambda=10000, \mu=0 \cdot 5, \beta=0 \cdot 005$.

\section{CONSEQUENCES OF NON-LINEAR \\ TRANSMISSION RATES AT THE \\ METAPOPULATION LEVEL}

\section{Metapopulation model with linear transmission rates}

Simulations of the spatially explicit model are in agreement with previous analyses, showing that for many combinations of host and nematode dispersal rates both host and parasite coexist, allowing the long-term persistence of the interaction (Fig. 3). Interestingly, time series generated from a single patch within the stable region (A) show that the local dynamics are highly unstable (Fig. 4a) akin to those seen for the entomopathogenic nematode non-spatial model described previously (see Fig. 2a) whereas the dynamics of the metapopulation as a whole (the sum of all patches) are buffered strongly at a very constant and stable level (Fig. 4b). Therefore, the explicit incorporation of space and localized, diffusive dispersal can have an important stabilizing effect on the host-parasite system.

\section{Metapopulation model with non-linear transmission rates}

Numerical simulations of the metapopulation model with non-linear transmission rates show the region of host-parasite coexistence to be larger than the corresponding region in the non-spatial model (Fig. 5). However, there is still a considerable region of parameter space where certain combinations of non-linear
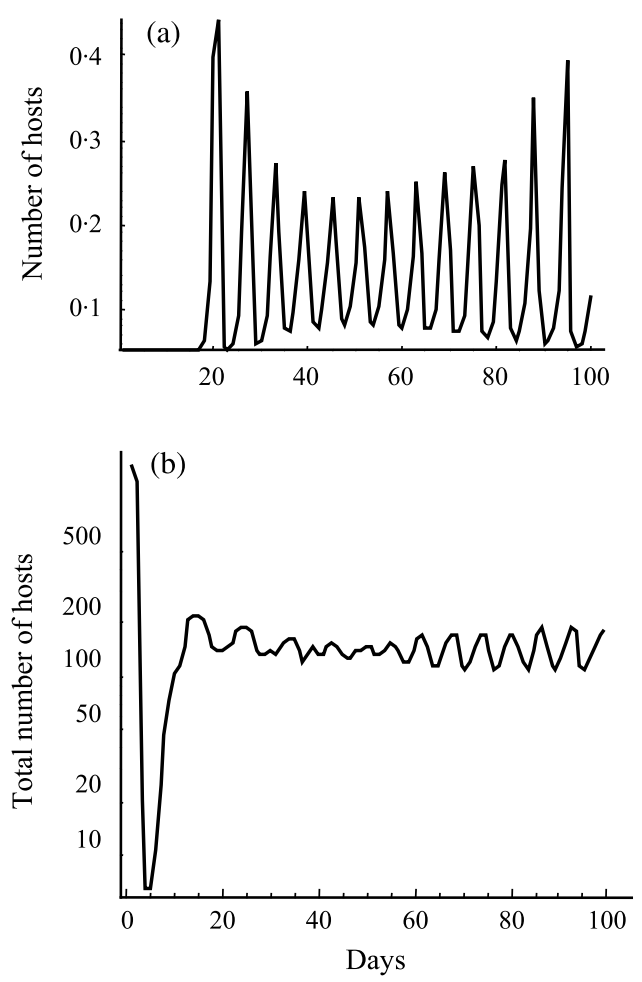

Fig. 4. Numerical simulations of the metapopulation model with linear transmission rates $[f(\mathrm{X}, \mathrm{P})=\beta \mathrm{XP}]$. (a) host numbers from a single patch within region A of Fig. $4\left(D_{H}=0 \cdot 2, D_{P}=\right.$ $0 \cdot 1)$ and (b) total host numbers, summed across the entire metapopulation. $r_{1}=0 \cdot 1, r_{2}=0, \gamma=0, b=0 \cdot 1, \alpha=0 \cdot 08$, $\Lambda=10000, \mu=0 \cdot 5, \beta=0 \cdot 005$.

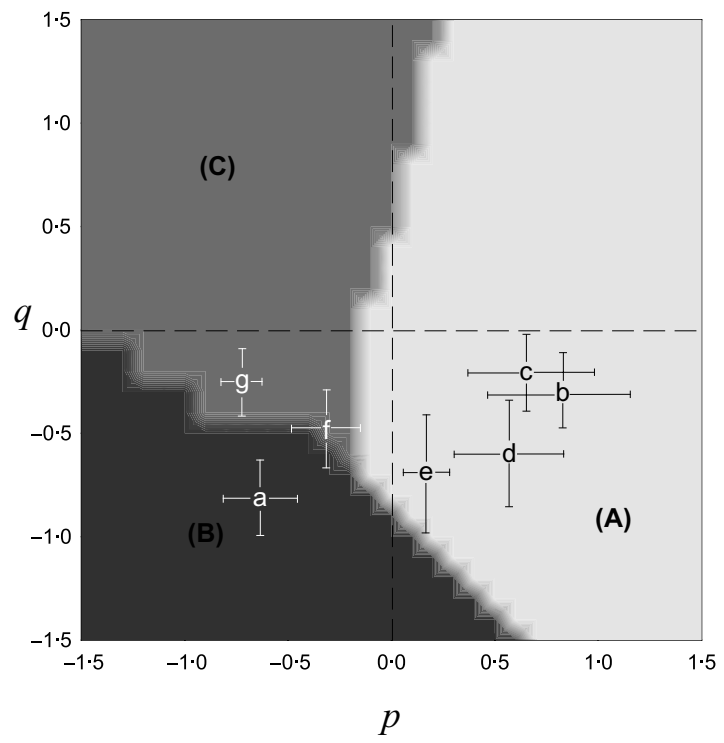

Fig. 5. $p-q$ parameter space showing regions of stability of the metapopulation model as obtained by numerical simulations of the model described in section $\mathrm{C} 2$ with non-linear transmission rates $\left[f(\mathrm{X}, \mathrm{P})=\beta^{\prime} \mathrm{X}^{p+1} \mathrm{P}^{q+1}\right]$. Region A represents stable host-parasite persistence, $\mathrm{B}$ represents regions where the parasite becomes extinct and $\mathrm{C}$ represents regions where the host becomes extinct. The lower case letters show the location of the host-parasite systems from Fig. 2. $r_{1}=0 \cdot 1$, $r_{2}=0, \gamma=0, b=0 \cdot 1, \alpha=0 \cdot 08, \Lambda=10000, \mu=0 \cdot 5, \beta^{\prime}=0 \cdot 145$, $\mathrm{D}_{\mathrm{H}}=0 \cdot 2, \mathrm{D}_{\mathrm{P}}=0 \cdot 1$. 
Unifying parasite transmission concepts
(C) 2002 British

Ecological Society, Journal of Animal Ecology, 71, 893-905 transmission rates (particularly when $p<0$ ) are sufficiently destabilizing to overcome the stabilizing influence of the explicit spatial effects and either the host or the parasites go extinct. Indeed, the regions of stability are qualitatively similar to those seen for the non-spatial model (compare Fig. 5 with Fig. 1), supporting the claims of Dwyer \& Elkinton (1993) that it may be the transmission processes at the local scale that drive epizootics observed at the metapopulation scale. This pattern is robust under a wide range of arena sizes and initial distributions of host and parasite. When we locate the $L$. dispar-NPV and $S$. feltiae-G. mellonella systems on Fig. 5 (points $a$ and $f$, respectively) we see that they still fall outside the region of stability implying, once again, that they should not be able to persist in the field. This finding is upheld regardless of the relative dispersal rates of the host or parasite or the duration of the period of interaction (not shown).

\section{Discussion}

In this paper we incorporated empirically derived estimates of parasite transmission rates with the appropriate model framework to determine how realistic transmission rates affect the dynamics and stability of host-parasite systems. It has been implied previously that non-linearities in transmission always stabilize dynamics (e.g. Barlow 2000) but we show that the influence of transmission rate is not clear cut; it cannot be assumed that just because transmission is non-linear with respect to host or parasite density it will always have a drastic influence on the behaviour of the system. In the case of entomopathogenic nematodes, for instance, the addition of a realistic transmission function does not increase stability; if anything, persistence is even less likely.

All of the parasites considered here, including the schistosome macroparasite, had transmission functions which decreased in response to increases in parasite density $(q<0)$, a form of transmission which has often been assumed for models of both host-parasitoid and host-pathogen systems (Gordon et al. 1991; Briggs \& Godfray 1995). Broadly speaking, two alternative hypotheses have been suggested to explain this phenomenon: saturation at high parasite densities or heterogeneities in the probability of infection (Knell et al. 1996; Dwyer et al. 1997; Knell et al. 1998a). Clearly, as parasite densities increase there must be a point at which saturation occurs and all the available hosts become infected. Hence per capita transmission rates decline with the addition of extra parasites as hosts become limiting (Knell et al. 1996). Alternatively, a negative relationship between parasite density and per capita transmission may be observed even at low densities if there is variability in the probability of contracting an infection. By adding an explicit heterogeneity term to the standard transmission model, Dwyer et al. (1997) showed that increasing the degree of heterogeneity among the host population leads to an increase in the degree of non-linearity in the transmission rate. Furthermore, Dwyer et al. (1997) showed that the heterogeneity model fitted the observed transmission rates better than the simple homogeneous model, attributing the heterogeneity to inherent variations in susceptibility among the hosts, including differences in susceptibility between instars (Dwyer 1991). However, such heterogeneities may also arise through an environmental or spatial factor which results in some hosts being more at risk of contacting the parasites than others (Knell et al. 1996, 1998a). It is not possible to come to any general conclusions about which mechanism is the most important and quite possibly both occur to a greater or lesser extent in all host-parasite systems. However, whatever the precise mechanism, it appears that as a general rule increases in the number of parasites always produce a reduction in the per capita rate of transmission.

When considering how transmission rates change with the density of susceptible hosts, the parasites studied here seem to fall into two distinct groups; those where transmission rates increased with host density $(p>0)$ and those with decreasing responses to host density $(p<0)$. The reason for this divide becomes clear when the biology of the organisms and especially the mode of transmission is considered. Infection by $B$. thuringiensis and PiGV tends not to result in host lysis; transmission occurs primarily through cannibalism of infected hosts by uninfected ones (Knell et al. 1996, 1998a). Therefore, at high densities, reduced food availability leads to increased cannibalism and increased transmission, resulting in stability of the system. It should be noted that this form of transmission resembles more closely direct transmission as assumed by Hochberg (1991) rather than the free-living infective stage model presented here, but the findings apply regardless of the details of the model; the observed non-linear transmission rates always have a stabilizing influence on the system.

Interestingly, Knell et al. (1998b) compared the dynamics of microcosms of $P$. interpunctella infected by either $B$. thuringiensis or PiGV. They found that $B$. thuringiensis typically became extinct whereas PiGV persisted throughout the duration of the experiment. The reason for this difference was attributed to the effect of susceptible host density on the basic reproductive ratio and the threshold host density for transmission, which was much lower for PiGV than for $B$. thuringiensis. It was shown that although these two pathogens transmit via similar, cannibalistic routes, this marked difference in dynamics arose due to subtle differences in the biology of the two systems. Bacillus thuringiensis appeared to have a strong antifeeding effect on $P$. interpunctella, leading to reduced cannibalism and therefore transmission (compare the $\beta^{\prime}$ values in Table 1 for Knell et al. (1996) and Knell et al. (1998b)). Indeed, in the analysis presented by Knell et al. (1998b), transmission of B. thuringiensis was found to be negative at low densities. Therefore, 
902

A. Fenton et al.

(C) 2002 British

Ecological Society,

Journal of Animal

Ecology, 71,

893-905 although per capita transmission increased with susceptible host density for $B$. thuringiensis, the effect was insufficient to overcome the very low, baseline rate of transmission at low densities, and the pathogen was driven to extinction. Hence, although non-linear transmission rates can have a strong influence on dynamics, it is important to consider other potential factors and especially other non-linearities in the biology of the system.

The remaining parasites examined here had decreasing transmission rates with increasing host density. This may be due, at least in part, to the behaviour of the hosts changing in response to density (Dwyer \& Elkinton 1993); in this case, susceptible hosts may be able to mix to a greater extent at low densities. Interestingly, however, the parasite systems with negative $p$-values tended to be the ones that infected via a free-living stage which, typically, moves at a lower rate than the host. Hence they are more likely to infect hosts that they are initially close to rather than those further away. Therefore, only subsets of the host and parasite populations mix homogeneously and the addition of extra susceptible hosts does not increase the number of hosts available for infection. Hence, per capita transmission rates decrease with increases in susceptible host density $(p<0)$.

This form of implicit spatial structuring, such that a fixed number of hosts are vulnerable to infection regardless of the total number within the arena, is reminiscent of how sexually transmitted diseases (STDs) are modelled. STDs are transmitted by very localized interactions whereby infected individuals mix with a relatively constant number of partners, regardless of the overall population size (May \& Anderson 1979; Thrall et al. 1993). As a result, STDs are modelled assuming frequency-dependent transmission using the function $\beta \mathrm{XY} / \mathrm{N}$ where $\mathrm{N}$ is the total population size so that as $\mathrm{N}$ increases the per capita transmission rate decreases in a manner similar to that described by $p<$ 0 in the Hochberg (1991) framework. Conversely, directly transmitted parasites which are transmitted by susceptibles and infecteds mixing at the same spatial scale are traditionally modelled using the densitydependent transmission function $\beta \mathrm{XY}$, akin to $p=$ $q=0$. It should be noted, however, that the terms frequency- and density-dependent transmission represent just two ends of a continuum of transmission types (Antonovics et al. 1995; McCallum 2000) and the pattern of transmission is likely to shift along this continuum according to the combination of densities studied and the scale of observation (Diekmann et al. 1995; Fromont, Pontier \& Langlais 1998). Following the above argument, this continuum can effectively be described by $p$ moving from negative to zero. It is gradually being realized that a greater range of parasites beyond just STDs (e.g. vector-borne diseases (Dye \& Hasibeder 1986), cowpox virus in bank voles (Begon et al. 1999), rabbit haemorrhagic disease (White et al. 2001) and the entomopathogenic nematodes described here) are transmitted in a manner more closely described by a frequency-dependent-type function due to transmission occurring within a socially or spatially structured framework.

It appears from the spatially explicit model that the nature of the transmission function at the local level can play a more important role in determining stability at the metapopulation level than the explicit incorporation of spatial structuring. While spatial effects and localized dispersal are generally thought to be stabilizing (Comins \& Hassell 1996; White et al. 1996), it was apparent that non-linear transmission could have a sufficiently negative effect to overcome the effects of space, destabilize the system and drive either host or parasite to extinction. This emphasizes the importance of correctly describing and quantifying the transmission function and we believe that the analytical approach described here, coupled with laboratory studies aimed at quantifying the nature of the transmission function at the individual level can be sufficient to provide a revealing insight into how a host-parasite system will behave at the population- or even metapopulation level (Dwyer \& Elkinton 1993).

From an applied point of view, it is interesting that the entomopathogenic nematodes sat in an unstable region of parameter space, suggesting they would become extinct regardless of any recovery effects through dispersal. Although this seems counter-intuitive, there are some data that support this prediction. Entomopathogenic nematodes are used as biological control agents of insect pests, often within glasshouses. Under these conditions, the nematodes are relatively successful at controlling the pest in the short term, but they often have to be reapplied to ensure control is maintained (Grewal \& Richardson 1993; Scheepmaker et al. 1997). Without repeated application in these closed one host-one parasite systems, the model predictions are upheld and the nematodes go extinct. However, it has been shown previously that the addition of a highly susceptible host into the glasshouse allows the nematodes to maintain themselves when pest densities are low, thereby enabling their persistence (Kaya 1990). It is only in natural populations in the field where there are other factors, including the presence of alternative hosts, that entomopathogenic nematodes have persisted for any length of time (Hominick \& Briscoe 1990; Parkman et al. 1993). It appears that these nematodes cannot be linked inextricably to a single host species and this may explain partly why they have such a vast host range.

This work reveals some of the diversity of parasite transmission functions that have been found in the past and shows how they affect the dynamics of the hostparasite system. The shape of the transmission function is a direct result of the biology of the host and parasite, meaning that it should be possible to develop a priori hypotheses as to the nature of the transmission function from a basic understanding of the life history of the organisms concerned. Clearly, this study has 
903

Unifying parasite

transmission

concepts considered only a limited range of parasite systems. It would be invaluable to determine the nature of the transmission function for a wide range of parasites, locate them within the framework described here and develop a more complete picture of how transmission affects the persistence of parasites in nature.

\section{Acknowledgements}

We thank Mike Boots, Rob Knell and an anonymous referee for their comments on earlier versions of this manuscript. This work was funded by a grant from NERC.

\section{References}

Anderson, R.M. \& May, R.M. (1979) Population biology of infectious diseases. Part I. Nature, 280, 361-367.

Anderson, R.M. \& May, R.M.(1981) The population dynamics of microparasites and their invertebrate hosts. Philosophical Transactions of the Royal Society of London, Series B, 291, 451-524.

Antonovics, J., Iwasa, Y. \& Hassell, M.P. (1995) A generalizedmodel of parasitoid, venereal, and vector-based transmission processes. American Naturalist, 145, 661-675.

Barlow, N.D. (2000) Non-linear transmission and simple models for bovine tuberculosis. Journal of Animal Ecology, 69, 703-713.

Begon, M., Feore, S.M., Bown, K., Chantrey, J., Jones, T. \& Bennett, M. (1998) Population and transmission dynamics of cowpox in bank voles: testing fundamental assumptions. Ecology Letters, 1, 82-86.

Begon, M., Hazel, S.M., Baxby, D., Brown, K., Cavanagh, R., Chantry, J., Jones, T. \& Bennet, M. (1999) Transmission dynamics of a zoonotic pathogen within and between wildlife host species. Proceedings of the Royal Society, Series B, Biological Sciences, 266, 1939-1945.

Beisner, B.E. \& Myers, J.H. (1999) Population density and transmission of virus in experimental populations of the western tent caterpillar (Lepidoptera: Lasiocampidae). Environmental Entomology, 28, 1107-1113.

Boots, M. (1999) A general host-pathogen model with freeliving infective stages and differing rates of uptake of the infective stages by infected and susceptible hosts. Researches on Population Ecology, 41, 189-194.

Briggs, C.J. \& Godfray, H.C.J. (1995) The dynamics of insectpathogen interactions in stage structured populations. American Naturalist, 145, 855-887.

Carter, N.P., Anderson, R.M. \& Wilson, R.A. (1982) Transmission of Schistosoma mansoni from man to snail: laboratory studies on the influence of snail and miracidial densities on transmission success. Parasitology, 85, 361372.

Comins, H.N. \& Hassell, M.P. (1996) Persistence of multispecies host-parasitoid interactions in spatially distributed models with local dispersal. Journal of Theoretical Biology, 183, 19-28.

Comins, H.N., Hassell, M.P. \& May, R.M. (1992) The spatial dynamics of host-parasitoid systems. Journal of Animal Ecology, 61, 735-748.

D'Amico, V., Elkinton, J.S., Dwyer, G., Burand, J.P. \& Buonaccorsi, J.P. (1996) Virus transmission in gypsy moths is not a simple mass action process. Ecology, 77, 201-206.
(C) 2002 British

Ecological Society, Journal of Animal Ecology, 71, 893-905
Dwyer, G. (1991) The roles of density, stage, and patchiness in the transmission of an insect virus. Ecology, 72, 559-574.

Dwyer, G. (1994) Density dependence and spatial structure in the dynamics of insect pathogens. American Naturalist, 143, 533-562.

Dwyer, G., Dushoff, J., Elkinton, J.S. \& Levin, S.A. (2000) pathogen-driven outbreaks in forest defoliators revisited: building models from experimental data. American Naturalist, 156, 105-120.

Dwyer, G. \& Elkinton, J.S. (1993) Using simple models to predict virus epizootics in gypsy moth populations. Journal of Animal Ecology, 62, 1-11.

Dwyer, G., Elkinton, J.S. \& Buonaccorsi, J.P. (1997) Host heterogeneity in susceptibility and disease dynamics: tests of a mathematical model. American Naturalist, 150, 685-707.

Dye, C. \& Hasibeder, G. (1986) Population-dynamics of mosquito-borne disease: effects of flies which bite some people more frequently than others. Transactions of the Royal Society of Tropical Medicine and Hygiene, 80, 69-77.

Fromont, E., Pontier, D. \& Langlais, M. (1998) Dynamics of a feline retrovirus (FeLV) in host populations with variable spatial structure. Proceedings of the Royal Society of London, Series B, Biological Sciences, 265, 1097-1104.

Gordon, D.M., Nisbet, R.M., DeRoos, A., Gurney, W.S.C. \& Stewart, R.K. (1991) Discrete generations in host-parasitoid models with contrasting life cycles. Journal of Animal Ecology, 60, 295-308.

Grewal, P.S. \& Richardson, P.N. (1993) Effects of application rates of Steinernema feltiae (Nematoda, Steinernematidae) on biological control of the mushroom fly Lycoriella auripila (Diptera, Sciaridae). Biocontrological Science and Technology, 3, 29-40.

Heesterbeek, J.A.P. \& Metz, J.A.J. (1993) The saturating contact rate in marriage and epidemic models. Journal of Mathematical Biology, 31, 529-539.

Hochberg, M.E. (1991) Non-linear transmission rates and the dynamics of infectious disease. Journal of Theoretical Bio$\log y$, 153, 301-321.

Hominick, W.M. \& Briscoe, B.R. (1990) Survey of 15 sites over 28 months for entomopathogenic nematodes (Rhabditida, Steinernematidae). Parasitology, 100, 289-294.

Kaya, H.K. (1990) Entomopathogenic nematodes in biological control of insects. In: New Directions in Biological Control: Alternatives for Suppressing Agricultural Pests and Diseases (eds R.R. Baker \& P.E. Dunn), pp. 189-198. Alan R. Liss, Inc., New York.

Knell, R.J., Begon, M. \& Thompson, D.J. (1996) Transmission dynamics of Bacillus thuringiensis infecting Plodia interpunctella: a test of the mass action assumption with an insect pathogen. Proceedings of the Royal Society of London, Series B, Biological Sciences, 263, 75-81.

Knell, R.J., Begon, M. \& Thompson, D.J. (1998a) Transmission of Plodia interpunctella granulosis virus does not conform to the mass action model. Journal of Animal Ecology, 67, 592-599.

Knell, R.J., Begon, M. \& Thompson, D.J. (1998b) Hostpathogen population dynamics, basic reproductive rates and threshold densities. Oikos, 81, 299-308.

Liu, W.M., Hethcote, H.W. \& Levin, S.A. (1987) Dynamic behavior of epidemiologic models with non-linear incidence rates. Journal of Mathematical Biology, 25, 359-380.

May, R.M. \& Anderson, R.M. (1979) Population biology of infectious diseases. Part II. Nature, 280, 455-461.

McCallum, H. (2000) Population Parameters: estimation for ecological models. Blackwell Science, Oxford.

McCallum, H., Barlow, N. \& Hone, J. (2001) How should pathogen transmission be modelled? Trends in Ecology and Evolution, 16, 295-300.

Parkman, J.P., Hudson, W.G., Frank, J.H., Nguyen, K.B. \& Smart, G.C. (1993) Establishment and persistence of
Reijnders, P. (1995) The force of infection in populations of varying sizes: a modelling problem. Journal of Biological Systems, 3, 519-529. 


\section{4}

A. Fenton et al.

Steinernema scapterisci (Rhabditida, Steinernematidae) in field populations of Scapteriscus spp mole crickets (Orthoptera, Gryllotalpidae). Journal of EntoMolecular Science, 28, 182-190.

Rohani, P. \& Miramontes, O. (1995) Host-parasitoid metapopulations: the consequences of parasitoid aggregation on spatial dynamics and searching efficiency. Proceedings of the Royal Society of London, Series B, Biological Sciences, 260, 335-342.

Scheepmaker, J.W.A., Geels, F.P., Smits, P.H. \& Van Griensven, L. (1997) Control of the mushroom pests Lycoriella auripila (Diptera: Sciaridae) and Megaselia halterata (Diptera: Phoridae) by Steinernema feltiae (Nematoda: Steinernematidae) in field experiments. Annals of Applied Biology, 131, 359-368.

Thrall, P.H., Antonovics, J. \& Hall, D.W. (1993) Host and pathogen coexistence in sexually-transmitted and vector-borne

\section{Appendix A}

\section{DETAILS OF THE METHODS USED TO ESTIMATE THE PER CAPITA TRANSMISSION COEFFICIENT, $\beta$, FROM EXPERIMENTAL DATA}

\section{General method for estimating $\beta$}

In all the studies described, the same basic approach is taken to estimate $\beta$. The initial number of hosts $\left(\mathrm{X}_{0}\right)$ and parasites $\left(\mathrm{P}_{0}\right)$ at the start of the experiment, the duration of the experiment, $t$, and the number of surviving hosts at the end of the experiment $\left(\mathrm{X}_{t}\right)$ is known, and it is assumed that hosts and parasites do not reproduce $(r=\Lambda \alpha=0)$. In the simplest case, as used by Knell et al. (1996), Knell et al. (1998a), and Beisner \& Myers (1999), parasite mortality is assumed to be negligible throughout the experiment $(\mu=0)$, so that the rate of change of the parasite population is assumed to be zero and $\mathrm{P}_{t}=\mathrm{P}_{0}$. The system can now be described by:

$\frac{d \mathrm{X}}{d t}=-\beta \mathrm{XP}_{0}$

By integrating the above equation with respect to $t$, the following expression is obtained:

$\beta=-\frac{1}{t \mathrm{P}_{0}} \ln \left(\frac{\mathrm{X}_{t}}{\mathrm{X}_{0}}\right)$

eqn A1

The experiment is conducted for a range of host and parasite densities and $\beta$ calculated for each combination. In the original papers the data is presented in the form of $\beta$ against density and from these data we estimate $\beta^{\prime}, p$ and $q$ from equation A4. In the following systems the approach differed from that described above due to different assumptions, primarily concerning the rate of loss of parasites throughout the experiment.

(C) 2002 British Ecological Society, Journal of Animal Ecology, 71, 893-905
Nuclear polyhedrosis virus and Lymantria dispar: D'Amico et al. (1996)

D'Amico et al. (1996) incorporated a term in their model to account for the decay rate of the pathogen $(\mu)$, diseases characterized by frequency-dependent disease transmission. American Naturalist, 142, 543-552.

White, A., Begon, M. \& Bowers, R.G. (1996) Host-pathogen systems in a spatially patchy environment. Proceedings of the Royal Society of London, Series B, Biological Sciences, 263, 325-332.

White, P.J., Norman, R.A., Trout, R.C., Gould, E.A. \& Hudson, P.J. (2001) The emergence of rabbit haemorrhagic disease virus: will a non-pathogenic strain protect the UK? Philosophical Transactions of the Royal Society, Series B, Biological Sciences, 356, 1087-1095.

Woods, S.A. \& Elkinton, J.S. (1987) Bimodal patterns of mortality from nuclear polyhedrosis virus in gypsy moth (Lymantria dispar L.) populations. Journal of Invertebrate Pathology, 50, 151-157.

Received 17 December 2001; revision received 7 May 2002

although they assumed the loss rate of virus particles through infection was insignificant. Therefore, their system was described by:

$\frac{d \mathrm{X}}{d t}=-\beta \mathrm{XP}$
$\frac{d \mathrm{P}}{d t}=-\mu \mathrm{P}$

leading to the following expression for $\beta$ :

$\beta=\frac{\mu}{\mathrm{P}_{0}\left(e^{-\mu t}-1\right)} \ln \left(\frac{\mathrm{X}_{t}}{\mathrm{X}_{0}}\right)$

eqn A2

\section{Entomopathogenic nematodes: Fairbairn et al. (unpublished)}

In this experiment, it was assumed that the rate of loss of nematodes through infection could have a significant effect on the rate of transmission. Hence, the rate of change of the nematode population throughout the experiment was not zero and the system was described by the equations:

$\frac{d \mathrm{X}}{d t}=-\beta \mathrm{XP}$

$\frac{d \mathrm{P}}{d t}=-n \beta \mathrm{XP}$

where $n$ was the mean number of nematodes infecting each host. In this case, integrating this system of equations and solving for $\beta$ leads to the more complex equation:

$\beta=\frac{-1}{t\left(\mathrm{P}_{0}-n \mathrm{X}_{0}\right)} \ln \left(\frac{\mathrm{X}_{t} \mathrm{P}_{0}}{\mathrm{X}_{0}\left(\mathrm{P}_{0}-n\left(\mathrm{X}_{0}-\mathrm{X}_{t}\right)\right)}\right)$ eqn A3

Schistosoma mansoni infecting snail intermediate hosts: Carter et al. (1982)

Carter et al. (1982) described the Schistosome-snail experimental system by:

$$
\begin{aligned}
& \frac{d \mathrm{X}}{d t}=-\beta \mathrm{XP} \\
& \frac{d \overline{\mathrm{P}}}{d t}=\beta \mathrm{XP}
\end{aligned}
$$


Unifying parasite transmission concepts where $\overline{\mathrm{P}}$ is the mean number of miracidial infections per snail (= P/X). Carter et al. (1982) argued that by assuming all snails have an equal probability of being infected by any one miracidium, the distribution of infections per snail would be positive binomial and so the proportion of infected snails $\left(\mathrm{I}_{t}=\mathrm{X}_{t} / \mathrm{X}_{0}\right)$ was:

$\mathrm{I}_{t}=\left[1-\left[1-\left[1-\exp \left(-\beta \mathrm{X}_{0} t\right)\right] / \mathrm{X}_{0}\right]^{\mathrm{P}_{0}}\right]$

which can be rearranged to produce the equation:

$$
\beta=\frac{-1}{\mathrm{X}_{0} t} \ln \left(1-\mathrm{X}_{0}\left(1-\left(1-\frac{\mathrm{X}_{t}}{\mathrm{X}_{0}}\right)^{\frac{1}{\mathrm{P}_{0}}}\right)\right)
$$

\section{Appendix B}

STABILITY ANALYSIS OF THE NON-SPATIAL HOST-MICROPARASITE MODEL WITH FREE-LIVING INFECTIVE STAGES AND LINEAR TRANSMISSION RATES

Setting equations A1-A3 equal to zero, with $f(\mathrm{X}, \mathrm{P})=\beta \mathrm{XP}$ and solving for $\mathrm{X}, \mathrm{Y}$ and $\mathrm{P}$ reveals two equilibria, the trivial solution $(0,0,0)$ and the nontrivial $\left(\mathrm{X}^{*}, \mathrm{Y}^{*}, \mathrm{P}^{*}\right)$ where:

$$
\begin{aligned}
\mathrm{P}^{*} & =\frac{A C}{\beta(C-B)} \\
\mathrm{X}^{*} & =\frac{\mu C}{\beta(\Lambda \alpha-C)} \\
\mathrm{Y}^{*} & =\frac{\mu A C}{\beta(C-B)(\Lambda \alpha-C)} .
\end{aligned}
$$

Clearly, for these equilibria to be biologically relevant, we need $\beta, A, C$ and $\mu>0$, and $\Lambda \alpha>C>B$. That is, the rate of production of new parasites needs to be greater than the rate of loss of infecteds which needs to exceed the rate of increase of the susceptible population as a result of the infecteds. The characteristic equation of the Jacobian evaluated at this equilibrium is:

$\lambda^{3}+\mathrm{x} \lambda^{2}+\mathrm{y} \lambda+\mathrm{z}=0 \quad$ where

$\mathrm{x}=C+\frac{A B}{(C-B)}+\mu+\frac{C \mu}{(\Lambda \alpha-C)}$

$\mathrm{y}=\frac{A \mu\left(B \Lambda \alpha-C^{2}\right)}{(C-B)(\Lambda \alpha-C)}$

$\mathrm{z}=A C \mu$.

and the $\lambda \mathrm{s}$ are the eigenvalues of the system. For the trivial solution $(0,0,0)$ the characteristic equation reduces to $(A-\lambda)(-C-\lambda)(-\mu-\lambda)=0$ which is always unstable because $A>0\left(r_{1}>b\right)$.

For the non-trivial solution, if the equilibria are biologically relevant, $x$ and $y$ are positive, satisfying two of the Routh-Hurwitz criteria for stability. Interestingly, the third criteria $(y>0)$ is upheld if $B \Lambda \alpha>C^{2}$. Therefore, it is essential that there is some form of increase in the susceptible population from the infecteds, either through recovery $(\gamma)$ or reproduction $\left(r_{2}\right)$ so that $B>0$.

\section{Appendix C}

STABILITY ANALYSIS OF THE NON-SPATIAL HOST-MICROPARASITE MODEL WITH FREELIVING INFECTIVE STAGES AND NON-LINEAR TRANSMISSION RATES

Using $f(\mathrm{X}, \mathrm{P})=\beta^{\prime} \mathrm{X}^{p+1} \mathrm{P}^{q+1}$ in equations $1-3$ and setting equal to zero reveals two equilibria, the trivial solution $(0,0,0)$ and the non-trivial $\left(\mathrm{X}^{*}, \mathrm{Y}^{*}, \mathrm{P}^{*}\right)$ where:

$$
\begin{aligned}
\mathrm{P}^{*} & =\left(\frac{\mu C}{\beta(\Lambda \alpha-C)}\left(\frac{\mu(C-B)}{A(\Lambda \alpha-C)}\right)^{-(p+1)}\right)^{\frac{1}{1+p+q}} \\
\mathrm{X}^{*} & =\frac{\mu \mathrm{P}^{*}(C-B)}{A(\Lambda \alpha-C)} \\
\mathrm{Y}^{*} & =\frac{\mu \mathrm{P}^{*}}{(\Lambda \alpha-C)} .
\end{aligned}
$$

As before, for this state to be biologically relevant, we need $\beta, A, C$ and $\mu>0$, and $\Lambda \alpha>C>B$. The characteristic equation of the Jacobian evaluated at this equilibrium is:

$$
\begin{aligned}
\lambda^{3}+ & \mathrm{x} \lambda^{2}+\mathrm{y} \lambda+\mathrm{z}=0 \quad \text { where } \\
\mathrm{x}= & \beta \mathrm{X}^{* p} \mathrm{P}^{* q}\left[\mathrm{P}^{*}(p+1)+\mathrm{X}^{*}(q+1)+C-A+\mu\right] \\
y= & \beta \mathrm{X}^{* p} \mathrm{P}^{* q}\left[\mathrm{P}^{*}(p+1)(\mu+C-B)-\right. \\
& \left.\mathrm{X}^{*}(q+1)(A+\Lambda \alpha-C)\right]+C(\mu-A)-A \mu \\
\mathrm{z}= & \beta \mathrm{X}^{* p} \mathrm{P}^{* q}\left[A \mathrm{X}^{*}(q+1)(\Lambda \alpha-1)+\right. \\
& \left.\mathrm{P}^{*} \mu(p+1)(C-B)\right]-A C \mu .
\end{aligned}
$$

and the $\lambda \mathrm{s}$ are the eigenvalues of the system. For the trivial solution $(0,0,0)$ the characteristic equation reduces to $(A-\lambda)(-C-\lambda)(-\mu-\lambda)=0$ which is always unstable because $A>0\left(r_{1}>b\right)$.

Although possible, an analytical solution of the Routh-Hurwitz criteria for the equilibrium ( $\mathrm{X}^{*}$, $\left.\mathrm{Y}^{*}, \mathrm{P}^{*}\right)$ is very complex and does not lead to any general insights. For the remainder of the analysis we resorted to numerical solutions to determine the boundaries of stability described in the main text. 